\title{
Funcionamiento ejecutivo en niños de educación infantil con diferentes niveles de matemáticas valorado por los maestros
}

José Antonio Rodríguez Niño jose.rodriguez@uji.es

María Llobet Piñas al202464@uji.es Lorena Zorrilla Silvestre al106987@uji.es 
I. Resumen

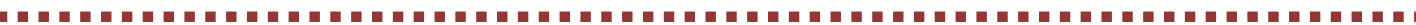

Algunos trabajos parecen apoyar la relación entre las funciones cognoscitivas o funcionamiento ejecutivo y el rendimiento matemático. Los problemas en este ámbito de funcionamiento en niños de etapa escolar han sido, en ocasiones, relacionados con déficits en memoria de trabajo, planificación o monitoreo. Con niños más pequeños, los estudios son muy escasos y discrepantes. El objetivo de este trabajo es profundizar en la relación existente entre diferentes factores de regulación conductual y metacognición de niños de Educación Infantil, con diferentes niveles de rendimiento matemático. Este estudio se ha realizado con 66 alumnos de la provincia de Castellón, de entre 5 y 6 años de edad, agrupados en dos grupos, buen nivel matemático y bajo nivel matemático. Los maestros cumplimentaron el cuestionario BRIEF (Behavior Rating Inventory of Executive Function: Gioia, Isquith, Guy y Kenworthy, 2000), un cuestionario muy útil para evaluar el funcionamiento ejecutivo de los niños. Los resultados muestran diferencias significativas entre los dos grupos evaluados en la mayoría de las áreas analizadas. Importantes implicaciones para el aprendizaje en matemáticas en la etapa de Educación Infantil pueden derivarse de estos resultados.

Palabras clave: funcionamiento ejecutivo, matemáticas, educación infantil, iniciativa, memoria de trabajo, planificación, monitorización, metacognición, regulación de conducta.

\section{Introducción}

A lo largo de la historia se ha observado que algunos niños presentan problemas en matemáticas durante su escolaridad y en su posterior vida adulta. Estas diferencias en la adquisición de las habilidades matemáticas provocan un retraso escolar, en ocasiones, significativo. Al igual que para la adquisición del proceso lector, son muchos los trabajos realizados en el campo del aprendizaje de las matemáticas, que reflejan la importancia de tres factores fundamentales en este proceso, a saber, las propias competencias matemáticas básicas, los factores motivacionales y el funcionamiento ejecutivo.

Diferentes planteamientos centrados específicamente en habilidades o competencias matemáticas básicas coexisten en la actualidad para la explicación de las dificultades de aprendizaje de las matemáticas. Desde el modelo piagetiano, la habilidad matemática depende del descubrimiento por parte del niño de una serie de operaciones lógicas básicas: seriación, clasificación, conservación e inclusión. Este planteamiento ha recibido el soporte de estudios que han mostrado la relación de estas operaciones lógicas con el rendimiento matemático del niño. 
Otros modelos, plantean que las operaciones lógicas no son condiciones previas, necesarias y suficientes para el aprendizaje de las matemáticas. Concretamente, desde una perspectiva neopiagetiana se considera que lo específico para el aprendizaje de la aritmética son las habilidades de conteo, que incluye tanto aspectos cognitivos -conceptual counting knowledge-como procedimentales - procedural counting knowledge.

Un enfoque alternativo y más reciente para explicar los problemas en el cálculo es el planteado por Butterworth (2003). Este autor, tras una revisión de trabajos sobre desarrollo infantil, neuropsicológicos, genéticos y de neuroimagen, concluye que la idea de numerosidad, entendida como la capacidad para estimar intuitivamente el número de elementos de un conjunto y sus relaciones -subitizing-, es la que mejor explica el desarrollo de las habilidades aritméticas y sus dificultades.

Por otro lado, diferentes investigaciones apuntan que las creencias atribucionales, o causas percibidas de éxito o fracaso de los niños, son una variable que tiene repercusiones directas en el rendimiento escolar general y específicamente en el área de las matemáticas. Las metas centradas en la tarea y centradas en el "yo» (Nicholls, 1989), las metas de aprendizaje y las metas de rendimiento (Ames, 1992) y los diferentes patrones motivacionales que desde modelos weinerianos adoptamos en función de cuáles sean las causas -locus de control, grado de estabilidad y grado de controlabilidad-, evidencian estas creencias atribucionales.

Por último, las funciones ejecutivas también han mostrado su importancia en el rendimiento matemático. Este dominio es en el que nos hemos centrado para realizar este trabajo. Las funciones ejecutivas son habilidades cognitivas, que guardan entre sí una relación. Incluyen habilidades vinculadas a la capacidad de organizar y planificar una tarea, seleccionar apropiadamente los objetivos, iniciar un plan y sostenerlo en la mente mientras se ejecuta, inhibir las distracciones, cambiar de estrategias de modo flexible si el caso lo requiere, autorregular y controlar el curso de la acción para asegurarse que la meta propuesta esté en vías de lograrse, etc.

En síntesis, inhibición, cambio, control emocional, iniciativa, memoria de trabajo, organización de materiales, monitorización y planificación y organización, constituyen habilidades importantes para resolver problemas de manera eficiente y eficaz.

Las funciones ejecutivas parecen ser fundamentales para el logro de metas escolares y laborales, ya que condicionan y organizan procesos cognoscitivos básicos, como son la memoria y la percepción requeridas para un comportamiento propositivo. Por este motivo, la alteración de estas funciones puede limitar la capacidad del individuo para mantener una vida independiente y productiva, aunque se encuentran intactas otras habilidades cognoscitivas.

Las investigaciones de Bull y otros (2008) y de Clark y otros (2010), han encontrado relaciones significativas entre las medidas de planificación, control inhibitorio, flexibilidad cognitiva y memoria de 
trabajo en último curso de preescolar, con el posterior rendimiento en matemáticas.

Así mismo, cabe destacar en este campo el trabajo de Toll y otros (2010), que utilizando un diseño longitudinal han encontrado que, entre un conjunto de funciones ejecutivas, las tres tareas de memoria de trabajo predecían la pertenencia al grupo de estudiantes con DAM, mientras que solamente una tarea de inhibición y ninguna de flexibilidad cognitiva demostraron el mismo poder predictor. Estos resultados informan de un poder explicativo superior de las funciones ejecutivas, especialmente de la memoria de trabajo, sobre los aprendizajes matemáticos.

\section{Objetivos}

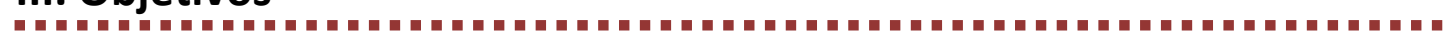

Teniendo en cuenta que: (a) el funcionamiento ejecutivo tiene peso en el aprendizaje de las matemáticas desde el inicio del desarrollo del niño y sobre todo en el futuro rendimiento; (b) no está claro qué dominios afectan con mayor intensidad en estas diferencias; y (c) que la mayoría de los estudios se han realizado mediante métodos de recogida de información en ambientes clínicos. El objetivo de este trabajo es realizar un análisis exhaustivo de las diferentes variables de funcionamiento ejecutivo que pueden afectar al rendimiento matemático en niños en edad preescolar según sus maestros. Para ello se utilizarán métodos de carácter ecológico, recogiendo información de los niños en contextos naturales, concretamente en el contexto escolar.

Teniendo en cuenta todos estos aspectos, la hipótesis de partida es que los niños con menor nivel matemático tendrán una mayor problematicidad en todas las variables de funcionamiento ejecutivo. Así mismo, se espera que la memoria de trabajo y al menos el control inhibitorio, la planificación y la organización de materiales tengan una mayor fuerza predictora de estas dificultades.

\section{Material y método}

En este estudio se ha utilizado una muestra de 66 niños (29 niñas y 37 niños) que estaban cursando el tercer trimestre de $3^{\text {er }}$ curso de Educación Infantil, de la provincia de Castellón, España, con una media de edad de 5 años y 10 meses. Los 8 colegios participantes, están repartidos en 3 localidades; 4 en Castellón capital, 3 en la Vall D’Uxó y 1 en Moncófar (7 públicos y 1 concertado).

Para evitar comprometer los resultados de este trabajo, los niños han sido seleccionados aleatoriamente. Con el fin de descartar aquellos niños con otras patologías, se les ha aplicado a todos dos subpruebas de la escala WIPPSI (escala de inteligencia de Wechsler para preescolar y primaria) que nos permite obtener un $\mathrm{Cl}$ equivalente. 
La muestra final se dividió en dos grupos, niños con un nivel normal/alto en matamáticas ( $n=35)$ y niños con un nivel matemático bajo $(n=31)$. Para realizar esta clasificación se utilizó un cuestionario cumplimentado por los maestros y las maestras, además de una prueba estandarizada de matemáticas que permite valorar las competencias matemáticas ya adquiridas, el TEDI-MATH de Grégoire y otros. Esta prueba consta de 25 subpruebas diferentes que valoran 6 ámbitos de conocimiento numérico.

El instrumento utilizado para la recogida de datos ha sido el BRIEF de Giogia, Isquith, Guy y Kenworthy (2000), el cual nos permite recoger por medio de 86 ítems la información necesaria de las diferentes variables de funcionamiento ejecutivo, en contextos naturales. Este cuestionario se rellena en menos de 15 minutos, y es de gran interés ya que ha sido utilizado en multitud de trabajos sobre este ámbito. Las variables de funcionamiento ejecutivo se recogen en dos grandes grupos, metacognición -iniciativa, memoria de trabajo, planificación/organización, organización de materiales y monitorización- y regulación de conducta -inhibición, cambio y control emocional-. Ofrece, además, una puntuación global o índice global compuesto, que recoge todas las funciones ejecutivas que se van a analizar en este trabajo. Informa también, sobre la consistencia/inconsistencia en la respuesta del maestro en el cuestionario y sobre la posible negatividad del evaluador hacia el evaluado, con el fin de descartar los cuestionarios que tuvieran estos sesgos.

El procedimiento seguido para la realización del trabajo de campo ha sido el siguiente: en primer lugar, dar información y realizar la petición formal tanto a los colegios como a Conserjería de Educación; en segundo lugar, nos hemos entrevistado con los docentes y hemos informado a los padres, que han autorizado a sus hijos para participar en el trabajo; en tercer lugar, hemos procedido con la selección de la muestra y la recogida de información por parte de la maestra y la entrega de los BRIEFs para los maestros.

A continuación se describen las variables de funcionamiento ejecutivo analizadas:

Memoria de trabajo

La memoria de trabajo, es la habilidad de retener en la mente aquello que se está realizando en este momento, qué he hecho, qué estoy haciendo y qué haré, como estructura básica a mantener en la memoria de trabajo durante la tarea. Una menor capacidad en esta habilidad, no solo puede provocar déficits matemáticos, sino que también se espera que pueda ocasionar dificultades a lo largo de toda la vida del sujeto.

La memoria de trabajo es una de las variables que se espera prediga en mayor medida las habilidades matemáticas. Trabajos como los de Anderson (2008), Bull y otros (2008), Passolunghi y otros (2007), y Toll y otros (2010), Vukovic y Siegel (2010) informan de que este dominio es el que mejor explica las dificultades en matemáticas, ya que para la resolución de cualquier problema matemático, retener en la memoria de 
trabajo todo un plan estratégico, de mayor o menor complejidad, es condición necesaria para una correcta resolución.

Planificación/Organización

En la misma línea que la memoria de trabajo, la elaboración de un plan de acción es tan sencilla como, en ocasiones, compleja. Para la ejecución de una tarea, parece casi imprescindible, cuando no se ha automatizado la práctica, la elaboración de un plan estratégico. Por tanto, para la matemática es necesario un plan para la resolución de problemas en tanto y cuanto para el cálculo aritmético. Defienden esta idea Bull y otros (2008), Brock y otros (2009) y Clark y otros (2010), estos últimos por medio de un estudio longitudinal, realizaron correlaciones entre los resultados de diferentes pruebas en varios grupos, concluyendo que la planificación es una de las habilidades que mayor correlación obtuvo.

\section{Organización de materiales}

Por otro lado, la organización de aquello que dispongo para la realización de una tarea, se espera que sea una habilidad que será más precisa en aquellos niños que muestren un nivel bueno en matemáticas. Ya que el desconocimiento, la desorganización, y en ocasiones la perdida de herramientas provoca que sea cuasi imposible alcanzar la meta esperada.

\section{Inhibición}

La inhibición, o capacidad para no atender a estímulos externos distractores o prestar atención a aquellos que son de interés para la ejecución de la tarea, nos parece que debe ser también un predictor de estas diferencias en matemáticas. Se espera que sea así a causa de la relación directa entre la capacidad de inhibición y las distracciones, un sujeto que se distrae con mayor facilidad, posiblemente encuentre dificultades mayores, por falta de concentración, mayor uso de la memoria, etc. Algunos estudios muestran la relación entre la inhibición y las dificultades matemáticas (Anderson, 2008; Brock y otros, 2009; Bull y otros, 2008; Clair-Thompson y Gathercole, 2006; Clark y otros, 2010; Toll y otros, 2010).

\section{Cambio}

Shifting, como es conocido por la mayoría de investigadores, es la capacidad de cambiar de una actividad a otra cuando la situación lo demande. Tras una revisión de diversas publicaciones a lo largo de este trabajo, la capacidad de cambio ha sido relacionada con las dificultades matemáticas por algunos autores (Anderson, 2008; Bull y otros, 2008) mientras que otros autores no encuentran dicha relación (Espy y otros, 2004; van der Sluis y otros 2004). 
Para realizar el análisis de resultados, hemos utilizado el programa informático IBM SPSS Statistics. Para comprobar la normalidad de los datos, se ha realizado la prueba de Kolmogorov-Smirnov. Una vez confirmada la normalidad de la distribución de todas las variables utilizadas, se han comparado las medias mediante la prueba Tstudent.

\section{Resultados}

Como se muestra en la Tabla 1 , los resultados indican que en el índice global compuesto (CEG) hay una diferencia importante entre las medias de ambos grupos, presentando mayor puntuación los niños y niñas del grupo de bajo nivel. Cabe destacar que en el BRIEF cuanto más alta es la puntuación mayor problematicidad habrá en el funcionamiento ejecutivo. Así mismo, en los índices globales de regulación de conducta y metacognición se observa una diferencia clara entre las medias siendo más elevadas las del grupo de bajo nivel.

Es importante destacar el hecho de que en cada una de las variables de funcionamiento ejecutivo analizadas, las medias de los sujetos que pertenecen al grupo de bajo nivel son, en todos los casos, más altas.

De esta forma, los resultados obtenidos a través de la prueba Tstudent muestra que es más alta la puntuación $\mathrm{t}$ del índice de metacognición, $\mathrm{t}=6.928$, frente a la del índice de regulación de conducta, $\mathrm{t}=2.852$, siendo la $\mathrm{t}$ más baja la del control emocional, $\mathrm{t}=1.671$. Así mismo, dos de las variables han obtenido mayor puntuación son planificación/organización, $\mathrm{t}=6.861$ y memoria de trabajo, $\mathrm{t}=6.760$. Finalmente, iniciativa, $t=7.385$, es la variable cuya puntuación T es la más elevada para ambos grupos.

En relación con los análisis estadísticos, observamos que, excepto en control emocional, los niños de ambos grupos presentan diferencias estadísticamente significativas en todas las variables analizadas.

Tabla 1. Comparación de los grupos con bajo y alto nivel matemático en funcionamiento ejecutivo

\begin{tabular}{|ll|c|c|c|c|}
\hline & GRUPO & Media & $\begin{array}{c}\text { Desviación } \\
\text { típ. }\end{array}$ & $\begin{array}{c}\text { Tstudent } \\
\text { (t) }\end{array}$ & $\begin{array}{c}\text { Significatividad } \\
\text { (bilateral) }\end{array}$ \\
\hline \multirow{2}{*}{ Inhibición } & Menor nivel & 17,23 & 5,433 & 2,901 &, $005^{* *}$ \\
& Mayor nivel & 13,80 & 4,136 & &, $006^{* *}$ \\
\hline \multirow{2}{*}{ Cambio } & Menor nivel & 15,03 & 3,851 & 2,872 &, 102 \\
\hline \multirow{2}{*}{ Control Emocional } & Mayor nivel & 12,57 & 2,993 & & \multirow{2}{*}{, $000^{* * *}$} \\
\hline \multirow{2}{*}{ Iniciativa } & Menor nivel & 13,74 & 5,489 & 1,671 & \\
\hline \multirow{2}{*}{ Memoria Trabajo } & Menor nivel & 11,91 & 2,801 & & \multirow{2}{*}{$000^{* * *}$} \\
& Mayor nivel & 8,86 & 2,746 & & \\
\hline
\end{tabular}




\begin{tabular}{|c|c|c|c|c|c|}
\hline $\begin{array}{l}\text { Planificación } \\
\text { Organización }\end{array}$ & $\begin{array}{l}\text { Menor nivel } \\
\text { Mayor nivel }\end{array}$ & $\begin{array}{l}18,29 \\
11,71 \\
\end{array}$ & $\begin{array}{l}4,656 \\
2,771 \\
\end{array}$ & 6,861 &, $000 * * *$ \\
\hline \multirow{2}{*}{ Organización Materiales } & Menor nivel & 9,87 & 3,354 & \multirow[t]{2}{*}{2,576} & \multirow[t]{2}{*}{, $013^{* *}$} \\
\hline & Mayor nivel & 8,03 & 2,281 & & \\
\hline \multirow{2}{*}{ Monitorización } & Menor nivel & 18,84 & 4,306 & \multirow[t]{2}{*}{6,653} & \multirow[t]{2}{*}{, $000 * * *$} \\
\hline & Mayor nivel & 12,86 & 2,713 & & \\
\hline \multirow{2}{*}{$\begin{array}{l}\text { Regulación Conducta } \\
\text { (IRC) }\end{array}$} & Menor nivel & 46,00 & 13,419 & \multirow[t]{2}{*}{2,852} & \multirow[t]{2}{*}{, $007 * * *$} \\
\hline & Mayor nivel & 38,29 & 7,262 & & \\
\hline \multirow{2}{*}{ Metacognición (IM) } & Menor nivel & 79,71 & 17,844 & \multirow[t]{2}{*}{6,928} & \multirow[t]{2}{*}{, $000 * * *$} \\
\hline & Mayor nivel & 53,06 & 12,588 & & \\
\hline \multirow{2}{*}{$\begin{array}{l}\text { Índice Global } \\
\text { Compuesto (CEG) }\end{array}$} & Menor nivel & 125,71 & 30,202 & \multirow[t]{2}{*}{5,604} & \multirow[t]{2}{*}{, $000 * * *$} \\
\hline & Mayor nivel & 91,34 & 16,925 & & \\
\hline
\end{tabular}

\section{Discusión y conclusiones}

Diferentes investigaciones apuntan la importancia del funcionamiento ejecutivo a lo largo del desarrollo y de la futura vida adulta (Sastre-Riba, 2006). Los resultados han ido siempre en esa dirección, es decir, funciones como el control conductual, la planificación, y la organización, entre otras, son habilidades fundamentales para la realización de cualquier tarea, por lo que los niños que tienen algún déficit en alguna de ellas puede tener problemas en su escolaridad. El funcionamiento ejecutivo ha sido investigado por muchos profesionales, en niños que presentan patologías diversas, informando de su trascendencia en niños y niñas con signos/riesgo o patologías ya diagnosticadas, como por ejemplo, los trabajos realizados por la Miranda (2001) y Miranda y otros (2005 y 2009).

Conocido el peso del funcionamiento cognoscitivo en la maduración y desarrollo del niño, se proponen investigaciones en el campo de las habilidades matemáticas. A través de estudios en su mayoría longitudinales, se ha dado luz a la importancia de esta habilidades ejecutivas en el aprendizaje y su aplicación en las matemáticas (Bull y otros, 2008; Espy y otros, 2004).

El objetivo de este trabajo era valorar si todas las habilidades de funcionamiento ejecutivo tienen el mismo peso y hay alguna variable que se asocie específicamente con los diferentes niveles de rendimiento matemática. Para muchos autores la respuesta es negativa. Véanse Bull y otros (2008), Clark y otros (2010) y Toll y otros (2010), que difieren es sus conclusiones.

Los resultados de este trabajo apuntan, en contra de lo esperado, que las habilidades inhibitorias, de cambio y de organización de materiales son las variables que menor capacidad discriminatoria tienen entre los grupos de alto y bajo nivel matemático.

Los dominios que explican en mayor medida estas diferencias entre grupos son memoria de trabajo, planificación/organización y 
monitorización. Sin embargo, la que diferencia especialmente a los grupos es la iniciativa, aquellos sujetos que menor puntuación han tenido en habilidades matemáticas son aquellos que muestran más dificultades en iniciar una tarea, independientemente de generar ideas.

En otro orden, el control emocional a pesar de ser la habilidad que menos diferencia a estos grupos muestra diferencias con un menor nivel significativo. Posiblemente originada por el tamaño de la muestra.

Finalmente cabe destacar, que entre los dos grandes grupos de habilidades cognoscitivas, regulación de conducta y metacognición, la que más predice los buenos resultados matemáticos es la metacognición. Además los resultados que hemos obtenido para el índice global compuesto son significativos, siendo los que mayor problematicidad presentan en funcionamiento ejecutivo aquellos niños y niñas que han participado como grupo de menor nivel en matemáticas.

Por tanto, podemos concluir que el funcionamiento ejecutivo es un predictor importante de las diferencias entre los diferentes niveles de rendimiento matemático. Estos resultados están en la misma línea de otros trabajos anteriores (Anderson, 2008; Bull y otros, 2008; Clark y otros, 2010).

Somos conscientes de que el tamaño de la muestra puede ser un factor influyente en los resultados, por lo que se invita a futuros estudios la utilización de muestras mayores que completara aquellos sesgos que como para el control emocional se pudieran haber creado. Así mismo, a pesar de lo concluido en este trabajo, cabe destacar que no solo el funcionamiento ejecutivo, como comentábamos con anterioridad, es un predictor de estas diferencias sino que también las variables motivacionales y las competencias matemáticas básicas lo son, por lo que deberían realizarse trabajos que incluyeran los tres factores.

Es interesante destacar que la aplicación de un programa que se incluya en el currículum el entrenamiento de las habilidades o funciones ejecutivas podría introducirse en la práctica docente. Un ejemplo de esta inclusión es el programa de Adele Diamond, Tools of the Mind que es un programa para la primera infancia basado en la investigación sobre la emergencia y la estimulación de las funciones autorregulatorias, a través de un currículo educativo de base vigotskiana. A través de una serie de rigurosas pruebas experimentales, Tools of the Mind -que recibió un premio de UNESCO a la excelencia como propuesta curricular-, ha demostrado tener un impacto significativo en el desarrollo de la autorregulación. El estudio también encontró que estas ganancias en la autorregulación, se asocian a mayores puntuaciones en el desempeño escolar durante las fases de alfabetización temprana y de adquisición de conocimientos matemáticos. 
VII. Bibliografía

AMES, C., (1992), "Classrooms: Goals, structures, and student motivation», Journal of Educational Psychology, Vol 84(3), Sep 1992, 261-271. doi: 10.1037/00220663.84.3.261.

ANDERSSON, U. (2008), "Working memory as a predictor of written Arithmetical skills in children: The importance of central executive Functions». British Journal of Educational Psychology, 78, 181-203.

BRoCK L., L. y otros (2009), "The contributions of 'hot' and 'cool' executive function to children's academic achievement, learning-related behaviors, and engagement in kindergarten", Early Childhood Research Quarterly, 24, Issue 3, 3rd Quarter, 337-349, ISSN 0885-2006, 10.1016/j.ecresq.2009.06.001.

BULL, R. y otros (2008), "Short-term memory, working memory, and executive functioning in preschoolers: Longitudinal predictors of mathematical achievement at age 7 years». Developmental Neuropsychology, 33, 205-228.

BUTTERWORTH, B (2003): Dyscalculia Screener, NFER-Nelson, London.

CLARK, C. A. C. y otros (2010), «Preschool executive functioning abilities predict early mathematics achievement». Developmental Psychology, 46(5), 1176-1191. doi: http://dx.doi.org/10.1037/a0019672

ESPY, K. A. y otros (2004), "The contribution of executive functions to emergent mathematic skills in preschool children». Developmental Neuropsychology, 26, 465-486.

MiRANDA CASAS, A. (2001): Trastornos por déficit de atención con hiperactividad: una guía práctica. Málaga: Aljibe.

Miranda CASAS, A. y otros (2005), «Nuevas tendencias en la evaluación de las dificultades de aprendizaje de las matemáticas. El papel de la metacognición». Revista de Neurología, 40, 97-102.

MiRANDA CASAS, A. y otros (2009), «Habilidades matemáticas y funcionamiento ejecutivo de niños con trastorno por déficit». Psicothema. Vol. 21, no 1, 63-69

PASSOLUNGHI, M. C. y otros (2007), "The precursors of mathematics learning: Working memory, phonological ability and numerical competence». Cognitive Development, 22, 165-184.

PiAget J. y R. GARCíA (1987): Hacia una lógica de significaciones. Barcelona: Gedisa.

Nicholls, J. G. (1984): Psychological Review. 
SASTRE-RIBA, S. (2006), "Condiciones tempranas del desarrollo y el aprendizaje: el papel de las funciones ejecutivas». Revista de neurología, 42 (Supl 2), S143-S151

St Clair-Thompson, H. L., y S. E. Gathercole (2006), «Executive functions and achievements in school: Shifting, updating, inhibition, and working memory». Quarterly Journal of Experimental Psychology, 59, 745-759.

TOLL, SYLKE W. M. y otros (2011), «Executive Functions as Predictors of Math Learning Disabilities». Hammill Institute on Disabilities. J Learn Disabil, 44, 521.

VAN DER SLUIS, S. y otros (2004), "Inhibition and shifting in children with learning deficits in arithmetic and reading». Journal of Experimental Child Psychology, 87, 239-266.

VUKOVIC, R. K. Y L. S. SIEGEL (2010), «Academic and cognitive characteristics of persistent mathematics difficulty form first through fourth grade». Learning Disabilities Research \& Practice, 25, 25-38. 
